\title{
Recent developments in pharmacoeconomic evaluation in Ireland
}

\author{
Expert Rev. Pharmacoeconomics Outcomes Res. 10(3), 221-224 (2010)
}

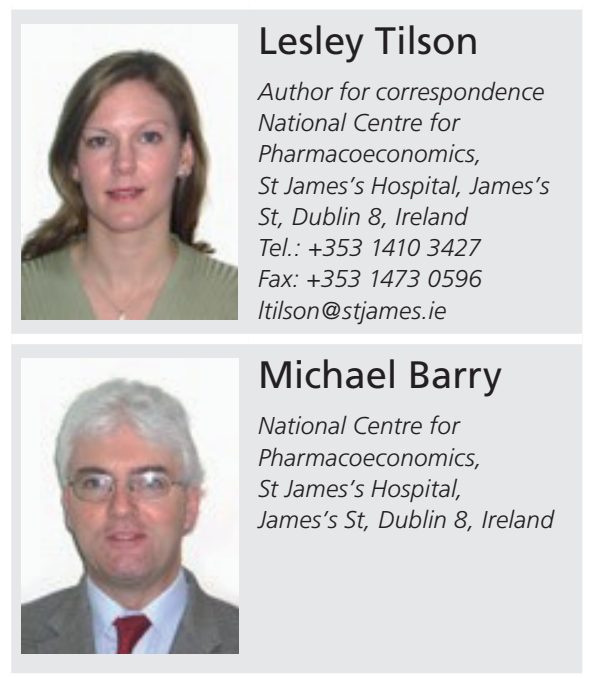

\begin{tabular}{l} 
EXPERT \\
REVIEWS \\
\hline
\end{tabular}

\author{
"The annual cost to the State of supplying medicines exceeds \\ $€ 2.24$ billion - a greater than sixfold increase has occurred over a \\ decade. This level of growth in expenditure is unsustainable."
}

Ireland experienced the largest population growth in the EU over the decade 19962006. Population growth is expected to continue and significant population aging is also predicted. A recent report, based on demographic and prescribing trends, estimated that the volume of prescriptions dispensed will continue to increase from 54 million items in 2006 to approximately 75 million in 2021 [101]. The annual cost to the State of supplying medicines exceeds $€ 2.24$ billion - a greater than sixfold increase has occurred over a decade. This level of growth in expenditure is unsustainable.

To ensure the continued provision of innovative and affordable medicines, various strategies have been introduced to ensure greater value for money from pharmaceutical expenditure. These strategies are described in the accompanying review article and include post-patent price cuts, increases in patient co-payments, and reductions in pharmacy and wholesale margins, as well as a formal requirement to demonstrate cost-effectiveness prior to the reimbursement of new medicines [1]. This editorial focuses on recent changes to the pharmacoeconomic process in Ireland as a result of the changing economic environment.

In September 2006, a formal requirement for evidence of cost-effectiveness in reimbursement decisions for certain medicines was introduced. High-cost products and those with a significant budget impact were subjected to formal pharmacoeconomic assessment. This represented a major change in the system for reimbursement of medicines in Ireland. More recent developments in the pharmacoeconomic evaluation of medicines in Ireland include: a rapid assessment of all new medicines, a dynamic cost-effectiveness threshold, an increasing importance of budget-impact assessment, conditional reimbursement and cost-effectiveness evaluation of existing medicines that are currently reimbursed.

\section{Pharmacoeconomic assessment of all new medicines}

Since September 2009, the National Centre for Pharmacoeconomics (NCPE), in collaboration with the Health Service Executive (HSE), have considered the cost-effectiveness of all new medicines following receipt of an application for reimbursement. In practice, all medicines are subjected to a preliminary rapid review. The rapid review process takes approximately 2-4 weeks and involves a review of the number of eligible patients in the population, the rate of uptake and whether the technology replaces or is added to current practice, the potential budget impact of the new technology, the price compared with the appropriate comparator(s) and clinical effectiveness, as well as cost-effectiveness data from other jurisdictions (if available). Products are then either recommended for reimbursement or referred for a formal pharmacoeconomic assessment. The formal pharmacoeconomic assessment is completed in less than 3 months.

\section{Cost-effectiveness threshold}

One of the implications of using costeffectiveness data in the decision-making process is that there is a threshold ratio above which an intervention would not be cost effective. In practice, there is no 
fixed cost-effectiveness threshold in Ireland. In the past, new drugs with an incremental cost-effectiveness ratio (ICER) below $€ 45,000$ /quality-adjusted life year (QALY) tended to be reimbursed. If a technology has an ICER that is significantly higher than other technologies that have been funded or reimbursed, other factors need to be considered. The following additional factors may be taken into account: the level of uncertainty associated with the clinical and cost-effectiveness information, budgetary considerations and the opportunity cost of investing in a certain intervention, the innovative nature of the technology and the lack of, or inadequacy of, alternative treatment options. For example, sunitinib (Pfizer, NY, USA) had an ICER of $€ 57,280 /$ QALY and was funded on the basis of its innovative nature [102]. If the ICER for a technology falls below that at which most interventions are funded, there is no guarantee of reimbursement and the level of uncertainty associated with the cost-effectiveness data plays an important part in the assessment.

"...the Minister for Health highlighted that, "we are showing what can be done by organizing ourselves better and getting the maximum value from the resources we have."

One of the requirements for the pharmacoeconomic submissions prepared by manufacturers for the NCPE is the inclusion of a probabilistic sensitivity analysis. This is considered an essential component of the evaluation to enable an assessment of the overall uncertainty in the evaluation. It enables the decision maker to interpret the results for a range of cost-effectiveness thresholds. The difference in price between the new drug and existing standard of care is also an important factor. The relationship between the price of the new product and the ICER is requested for the one-way sensitivity analysis. The recent NCPE evaluation of agomelatine for the treatment of major depression highlights the importance of the sensitivity analysis in the evaluation process. The probability that agomelatine would be cost effective compared with venlafaxine was $83 \%$ at a threshold of $€ 45,000 /$ QALY but only $30 \%$ if a threshold of $€ 20,000 / Q A L Y$ was applied [103]. Furthermore, the price of agomelatine was significantly higher than its comparators. Owing to the relatively high price and level of uncertainty identified by the cost-effectiveness evaluation, the drug received a negative assessment and was only reimbursed following a price reduction.

Bridges et al. recently raised the issue of whether a fixed cost-effectiveness threshold should be maintained at all and put forward arguments for and against a fixed threshold from a US perspective [2]. The benefits for a fixed threshold include its intuitive appeal for policy makers and the need for consistency and transparency. A fixed threshold provides the manufacturer of a new technology with an understanding of what is required for achieving reimbursement. On the other hand, the rigidity of a fixed threshold means that there may not be a case for exceptions to be made. Arguments against a fixed threshold include the imperfections of the piecemeal evaluation approach, which fails to assess the allocation of a budget across all interventions and impractical assumptions made in the application of a cost-effectiveness analysis; for example, should the same threshold apply to so-called lifestyle drugs and orphan drugs? What if a program is only partially implemented?

\section{Affordability of cost-effective interventions}

The issue of affordability of a cost-effective technology has recently arisen with the assessment of the human papilloma virus vaccine (HPV). The ICER for vaccinating 12-year-old girls against HPV 16 and 18 was $€ 17,383 /$ life-year gained, with the cost of the HPV vaccine plus administration costs in the first year of vaccination estimated at $€ 9.7$ million. The corresponding figures for vaccinating 12-year-old girls plus the catch-up to 15-year-old girls resulted in an ICER of $€ 52,968 /$ life-year gained and a budget impact of $€ 38.9$ million in the first year of vaccination $[3,104]$. In August 2008, vaccination of 12-year-old girls was approved and the catch-up programs were rejected due to budgetary constraints and competing demands for funding. Owing to the serious and rapid decline of the economic situation in Ireland later that year, the introduction of the vaccination program was delayed. In 2010, HPV vaccination became one of three top priority interventions for the Cancer Control Program after substantial price reductions for the vaccine were negotiated. HPV vaccination is to be introduced in Ireland later this year for approximately 30,000 girls who are in their first year of secondary school. Speaking at the time of this announcement about cancer services, the Minister for Health highlighted that, "we are showing what can be done by organizing ourselves better and getting the maximum value from the resources we have". The outcome in relation to HPV vaccination indicates that while the incremental cost-effectiveness of a technology is an important consideration, budget impact may have a greater influence over reimbursement decisions.

\section{"... it is evident that we are facing new challenges in funding very high-cost medicines with insufficient evidence to prove cost-effectiveness."}

\section{Conditional reimbursement}

The accompanying special report in this issue of Pharmacoeconomics and Outcomes Research by Barry et al. highlights that the growth in expenditure on the High Tech Drugs Scheme is a real cause for concern for the healthcare payer [1]. This scheme facilitates the reimbursement of high-cost drugs in primary care and is coming under increasing pressure with the emergence of very high-cost drugs, such as eculizumab (Soliris ${ }^{\circledR}$, Alexion Pharmaceuticals, CT, USA) - the terminal complement inhibitor for the treatment of paroxysmal nocturnal hemoglobinuria. The prevalence of paroxysmal nocturnal hemoglobinuria is 15.9 individuals per million. Eculizumab costs over $€ 350,000$ per patient per year and the estimated annual budget impact is at least $€ 3.5$ million per year, assuming that $15 \%$ of the eligible population would receive eculizumab [105]. Although manufacturers indicate that the patient population for such medicines is small, it is evident that we are facing new challenges in funding very high-cost medicines with 
insufficient evidence to demonstrate cost-effectiveness. Following a pharmacoeconomic assessment, eculizumab was not reimbursed under the High Tech Drugs Scheme and is restricted to prescribing in a specific hospital center. The details of the funding arrangements for this drug remain to be established.

There is a growing international interest in the potential for risk sharing and performance-based agreements, and this topic has been the subject of recent review articles in the literature. There are many different 'terms of arrangements' for these schemes, which may be based on health-outcome or financial-outcome guarantees. The literature highlights the need for 'best practice' guidelines regarding the implementation, governance and evaluation of such schemes, as the challenges involved in negotiating the terms of these agreements can be complex and resource intensive. A recent review highlighted that, although various approaches have been experimented with, many of them have not achieved the desired goal and many issues remain unresolved [4]. In Ireland, we anticipate that in exceptional circumstances, payers may turn to performance-based or risk-sharing agreements in an attempt to contain costs and minimize risks while enabling patients to gain early access to new very high-cost drugs. To date, the HSE has tended to adopt a pragmatic approach to negotiating price reductions prior to reimbursement if major uncertainty exists in relation to the cost-effectiveness of a new technology. The balance between providing access to new technologies and acknowledging that some degree of uncertainty will always exist regarding the clinical and cost-effectiveness of a technology is an ongoing challenge for healthcare payers.

\section{Disinvestment: new drugs for old}

All countries are facing the dilemma of how to maintain and improve quality of care in the face of static or shrinking budgets. Hughes et al. recently published an article about disinvestment and NICE, and highlighted that the funding of new, expensive medicines relies increasingly on releasing funds by displacing other treatments [5]. In Ireland, it was suggested in a report prepared on behalf of the Department of Health and Children that more focus should be applied to identifying topics for potential disinvestment so that ineffective and inefficient products and practices do not remain in the health system, and to free up resources for innovative medicines [106].

Glucosamine represents the first existing medicine to be selected for pharmacoeconomic assessment, subsequent to reimbursement having already been granted. In June 2009, the NCPE completed the pharmacoeconomic appraisal of glucosamine and did not recommend it as a cost-effective use of resources in the Irish healthcare setting [107]. State expenditure on glucosamine exceeds $€ 10$ million annually, which is substantially higher than the budget impact of the majority of new drugs seeking reimbursement. Reimbursement of glucosamine on the Community Drug Schemes is currently under review.

\section{"There is a growing international interest in the potential for risk sharing and performance-based agreements..."}

With a reduction in the healthcare budget of approximately $€ 1$ billion in 2010, access to new drugs will depend on savings found elsewhere within the healthcare system. In the future, if inefficient treatments are to be discontinued in Ireland, it is important that a consistent systematic approach is adopted regarding decisions on which treatments to discontinue. Such an approach would provide an important means of freeing up resources to enable healthcare funding to be focused on interventions that optimize health outcomes.

\section{Conclusion}

Since the economic downturn and cuts to the healthcare budget, there have been further changes to the pharmacoeconomic process in Ireland. The key changes include the rapid assessment of all new medicines seeking reimbursement, a dynamic cost-effectiveness threshold, an increasing importance of budget impact assessments, conditional reimbursement and the pharmacoeconomic assessment of existing medicines. In the future, we anticipate further downward pressure on drug expenditure and growing challenges for healthcare payers to fund very high-cost medicines with insufficient evidence to prove cost-effectiveness.

\section{Financial \& competing interests disclosure}

The authors have no relevant affliations or financial involvement with any organization or entity with a financial interest in or financial conflict with the subject matter or materials discussed in the manuscript. This includes employment, consultancies, honoraria, stock ownership or options, expert testimony, grants or patents received or pending, or royalties.

No writing assistance was utilized in the production of this manuscript.

\section{References}

1 Barry M, Usher C, Tilson L. Public drug expenditure in the Republic of Ireland. Expert Rev. Pharmacoeconomics Outcomes Res. 10(3), 239-245 (2010).

2 Bridges J, Onukwugha E, Mullins D. Healthcare rationing by proxy. Cost-effectiveness analysis and the misuse of the $\$ 50,000$ threshold in the US. Pharmacoeconomics 28, 175-184 (2010).
3 Usher C, Tilson L, Olsen J et al. Cost effectiveness of $\mathrm{HPV}$ vaccine in reducing the risk of cervical cancer in Ireland due to HPV types $16 \& 18$ using a transmission dynamic model. Vaccine 26, 5654-5661 (2008).

4 Stafinski T, McCabe C, Menon D. Funding the unfundable. Mechanisms for managing uncertainty in decisions on the introduction of new and innovative technologies into healthcare systems. Pharmacoeconomics 28, 113-142 (2010).
5 Hughes D, Ferner R. New drugs for old: disinvestment and NICE. BMJ 340, C572 (2010).

\section{Websites}

101 Layte R, Barry M, Bennett K et al. Projecting the impact of demographic change on the demand for and delivery of healthcare in Ireland. Research series number 13 (2009) 
www.esri.ie/UserFiles/ publications/20091023164031/RS013.pdf (Accessed March 2010)

102 National Centre for Pharmacoeconomics. A review of the cost-effectiveness of sunitinib $\left(\right.$ Sutent ${ }^{\circledR}$ ) under the High Tech Drug Scheme in Ireland. October 2006 www.ncpe.ie/u_docs/doc_129.pdf (Accessed April 2010)

103 National Centre for Pharmacoeconomics. Economic evaluation of agomelatine $\left(\right.$ Valdoxan $^{\circledR}$ ) for the treatment of major depressive disorder. September 2009 www.ncpe.ie/u_docs/doc_176.pdf (Accessed April 2010)
104 Health Information and Quality Authority. Health technology assessment of the role of human papillomavirus vaccines in reducing the risk of cervical cancer in Ireland (2008) www.hiqa.ie

(Accessed March 2010)

105 National Centre for Pharmacoeconomics. An economic evaluation of eculizumab (Soliris ${ }^{\circledR}$ ) for the treatment of paroxysmal noctural haemoglobinuria $(\mathrm{PNH})$ in the Irish healthcare setting. March 2010 www.ncpe.ie/u_docs/doc_188.pdf (Accessed March 2010)
106 Department of Health and Children. Economies in drug usage in the Irish healthcare setting (2009) www.dohc.ie (Accessed June 2009)

107 National Centre for Pharmacoeconomics. Economic evalution of glucosamine sulfate $\left(\mathrm{DONA}^{\circledR}\right)$ for the treatment of oestoarthritis in the Irish Healthcare setting. June 2009

www.ncpe.ie/u_docs/doc_171.pdf (Accessed March 2010) 